\title{
ARDS in radiation induced lung injury and Covid- 19 pneumonia: two different sides of the same coin. A
} case series comparison

\begin{abstract}
Coronavirus pneumonia (Covid-19 pneumonia) is a highly severe acute syndrome due to coronavirus 2 (SARS-CoV-2) infections, which has rapidly spread to other countries beyond China resulting in a global pandemic. Severe lung disease onset might result in death due to massive alveolar damage and severe progressive respiratory failure leading to acute lung injury which predispose to severe adult respiratory distress syndrome (ARDS). Symptoms, chest CT scan features and autopsy reports by a radiation oncologist's point of view, bring to mind a similarity with pictures of sporadic acute fatal radiation induced lung injury (RILI) which is a side effect of thoracic radiotherapy. Like two sides of the same coin, there is the feeling of a common inflammatory response of lung to an injury whatever it may be, virus, drugs or ionizing radiation as we found in these two case reports.
\end{abstract}

Volume II Issue 5 - 2020

\section{Grazia Lazzari, Giovanni Silvano}

Radiation Oncology Unit, San Giuseppe Moscati Hospital, Taranto, Italy

\author{
Correspondence: Grazia Lazzari, Radiation Oncologist, \\ Radiation Oncology Unit, San Giuseppe Moscati Hospital, \\ Roadto Martina Franca, 74100 Taranto, Italy, \\ Tel +390994585 I80, Email lazzaigrazia@gmail.com
}

Received: October 01, 2020 | Published: October 19, 2020

Keywords: SARS-CoV-2, lung injury, RT-PCR, DAMPs, PUMPs

\section{Introduction}

Acute respiratory distress syndrome (ARDS), is a syndrome of acute hypoxemic respiratory failure due to alveolar-capillary damage resulting from a variety of direct and indirect injuries to the gas exchange parenchyma of the lungs. ${ }^{1,2}$ Bacterial or viral infections, barotraumas, chemicals or ionizing radiation are the main responsible factors. However, regardless the cause, inflammation and alveolar damage are the main events. Respiratory distress symptoms requiring ventilation protocols in intensive care together with systemic phenomenon including thrombotic events such as DIC and multiple organ failure are accounted. ARDS has been widely described in Covid-19 (Co-19) fatal pneumonia, ${ }^{3}$ providing several features that bring to mind the characteristics of sporadic acute radiation induced lung injury (RILI).

It consists of a massive inflammatory involvement of both lungs occurring a few weeks after a limited field thoracic radiotherapy caused by a hypersensitivity immune mechanism triggered by radiation. ${ }^{4}$ Unlike the classical radiation pneumonitis, the sporadic one shows lung inflammatory symptoms with radiological findings occurring outside the radiation fields, making the differential diagnosis difficult with Co-19 pneumonia as we described in these two ARDS case reports. Swab, real time- PCR- test (RT -PCR-) and bronco-alveolar lavage (BAL) fluid were mandatory to improve differential diagnosis.

\section{Sporadic RILI fatal case}

This patient was a 70-year-old man who had a lung cancer in the right lung treated with lobectomy and adjuvant chemotherapy followed by postoperative radiotherapy. He received 50Gy on limited field of right mediastinum and hilum with 3D conformal EBRT. The analysis of treatment plan showed a safe dose-volume histogram (DVH) accounting a V5, a mean lung dose (MLD) and V20 in the normal range.

One-month radiotherapy off, he returned to our observation complaining of a cough, a fever of $39^{\circ}$, fatigue and worsening dyspnea. At admission, saturation $\mathrm{O}_{2}$ was $<70 \%$, worsening to require
$\mathrm{C}-\mathrm{PaP}$ and ventilation protocol; thus he was admitted to an intensive care unit.

Anemia, lymphopenia, neutrophilicleucocytosis were firstly assessed; in turn piastrinopenia with high levels of D-Dimer and fibrinogen were recorded. The Il-6 value was over the normal range. Sputum was negative for opportunistic.Swab, RT-PCR and BAL fluid were negative for SARS-CoV-2. BAL fluid showed inflammatory cells with altered clung cells, neutrophil, macrophages and activated lymphocytes. The CT images of the chest, described within surgical scarring of the right lung, a pleural effusion with air bronchogram and a massive bilateral alveolar thickening with diffuse ground-glass opacities evolving into a whited out lung (Figure 1).

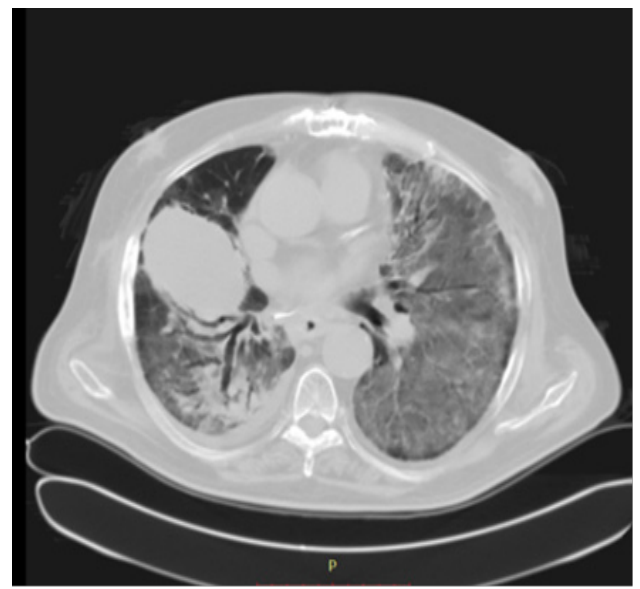

Figure I Chest CT scan of RILI case showing the whited out lung started from the irradiated right lung.

By autopsy, massive alveolar disruption with micro vascular hemorrhage, microthrombi and hyaline membranes were observed due to intra-alveolar fibrin deposits and serous fluid. At microscopy, pneumocytes exfoliation, platelets clots, trapped neutrophil and loads of giant macrophages were found. A diagnosis of sporadic RILI due to Hypersensitivity pneumonitis was done. 


\section{Covid-I 9 pneumonia fatal case}

This patient was a 65 -year-old man who was admitted to emergency unit in our hospital because he complained of fever refractory to antibiotics, acute severe dyspnea and cough. Saturation $\mathrm{O}_{2}$ was $60 \%$, ERS and D-Dimer title and IL-6 were over the normal range. Swab was positive for SARS-CoV-2. The patient underwent C-pap and intensive ventilation protocol in intensive care unit. The $\mathrm{CT}$ images of the chest recorded many diffuse bilateral ground-glass opacities, air bronchogram and interstitial thickening involvement evolving into a whited out lung (Figure 2).

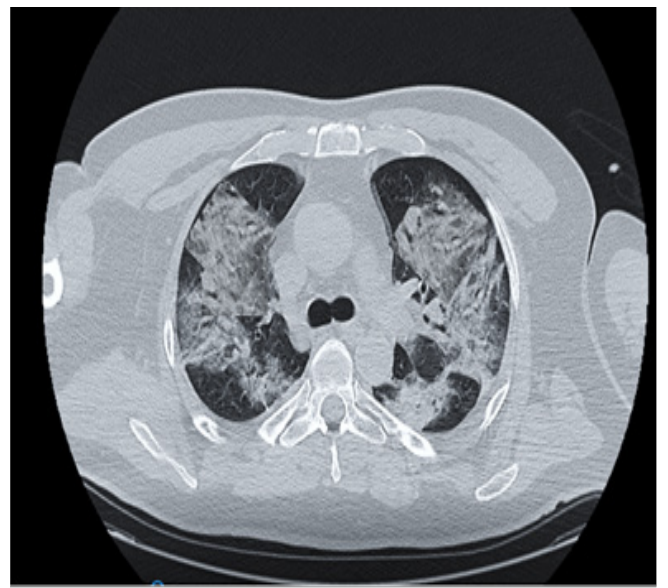

Figure 2 Chest CT scan of Co-19 pneumonia case showing the initial evolution into a bilateral whited out lung.

Two days later he died due to ARDS evolution. The autopsy revealed a massive bilateral lung injury with alveolar exudative inflammation, interstitial inflammation and focal pulmonary bleeding. In particular, the alveolar spaces appeared destroyed and showed a diffuse pneumocytes exfoliation with piles of cellular stromas and inflammatory cellular load. The alveolar capillaries were dilated and congested, the interstitium and the alveolar wall appeared thicker and transparent due to jaline membranes. Thrombosis into the microvessels were recorded with some areas of interstitial fibrosis. The oozing cells in the alveolar cavity were mainly monocytes, giant macrophages and neutrophylis trapped into platelets clots. Virus was detected in the alveolar cells. A diagnosis of fatal Co-19 pneumonia was made.

\section{Discussion}

Although causes were different, clinical pictures with ARDS as a final outcome were similar in both cases. In RILI dry cough, dyspnea, fever $38^{\circ}-39^{\circ}$ have been described within a few weeks after the completion of radiotherapy in almost all of the irradiated patients. ARDS is a rare event which is reported in cases of sporadic RILI. Pyrexia and respiratory distress symptoms are present in patients with Co-19 pneumonia in $85 \%$ of cases during their illness course where a more severe insult on the lung tissue might result in ARDS which can further precipitate septic shock and massive diffuse thrombosis. ${ }^{2}$ Haematological abnormalities like anemia or piastrinopenia or decreased lymphocyte count together to neutrophilia are accounted as in RILI than in Co-19 pneumonia. The thrombogenic biomarker D-dimer may become increased with a systemic elevation of the pyrogenic cytokines such as IL-6, IL-10, and TNF- $\alpha$ in both situations. ${ }^{5,6}$

Although chest CT scans play a key role in detecting and monitoring the pneumonia with a $98 \%$ sensitivity in Co -19 patients with false negative RT-PCR results, these findingshave been defined not specific for Covid-19 pneumonia diagnosis. In fact, CT scans are able to detect also RILI in all its phases and evolution over time with different patterns according the severity and phase of evolution as observed in Co -19 pneumonia. Findings like a combination of homogeneously increased density and patchy consolidation, diffuse ground glass opacities are the most reported signs in both diseases. ${ }^{7,8}$ In particular, in sporadic RILI, patterns of CT findings are spread in both lungs outside the radiation ports providing the image of a "whited out lung". This picture is also described in Co-19 pneumonia due to a massive lung involvement. ${ }^{9}$ Further, similarities can be found in autopsy reporting.

A series of open-lung biopsies taken from within the field of irradiation in patients with acute-phase RILI have shown neutrophil load with a large amount of mononuclear cells within the destroyed alveolar spaces and a lymphocytic infiltration within an edematous pulmonary interstitium. ${ }^{10}$ Findings such as hyaline membranes, marked cytological atypia within hyperplastic and exfoliated pneumocytes, and prominent vascular changes are also present with clots and microthrombi in the vessels in both lungs. With regard to Co-19 pneumonia, the inflammatory nature of lung damage by SARS$\mathrm{CoV}-2$ has been reported so far. In fact, histological examinations from lungs of deceased patients have shown bilateral diffuse alveolar damage with cellular fibromyxoid exudates. In the case of ARDS a massive desquamation of pneumocytes and hyaline membrane formation have been found in both lungs. Pulmonary oedema with hyaline membrane formation, interstitial mononuclear inflammatory infiltrates, dominated by lymphocytes and neutrophilis have been assessed. Diffuse clot of platelets and trapped neutrophylis have been also described. Given these similarities, these two cases seem different sides of the same coin as the lung response damage to an injury could be. ${ }^{11}$

It is acknowledged that RILI is driven by the direct cytotoxic action of ionizing radiation on lung cells activating a self-sustaining inflammatory cascade. A hypersensitivity effect may amplify the alveolar damage from the irradiated to the contralateral healthy lung as occurs in sporadic acute RILI. This event is a rare acute phenomenon occurring in $10 \%$ of cases a few weeks after limited field thoracic radiotherapy, involving both lungs with ARDS as a final outcome. ${ }^{12}$ As a mechanism, ionizing radiation generates tissue reactive free radicals (ROS) that can damage DNA molecules and cause clonogenic death in alveolar epithelial cells through the release of damage-associated molecular patterns (DAMPs), irrespective if the dying cells are malignant or not.

These molecular dysregulations and the lethality of ionizing radiation to type 1 pneumocytes, alveolar epithelial and endothelial cells provide the loss of barrier function and its structural denudation to promote recruitment of inflammatory cells, activated alveolar macrophages and platelets, followed by various activations of signal transduction pathways. These processes lead to the replacement of damaged cells, influx of inflammatory cells from peripheral blood and cytokine production, uncontrolled matrix turnover, and aberrant repair evolving into ALI and then ARDS. ${ }^{13}$

In sporadic RILI, the hypersensitivity theory has been postulated. Hypothesis is a possible direct tissue damage from radiotherapy which causes an amount of tissue antigen release,,$^{14}$ overcomes the normal tolerance processes and produces sensitization of autoreactive lymphocyte clones. In turn they migrate to the lung and react with pulmonary tissues leading to a massive bilateral inflammatory lung involvement. Regarding the Co-19 pneumonia, it has been speculated 
that SARS-CoV-2 particles invade the respiratory mucosa firstly and then infect other the alveolar cells, triggering a damage to the barrier with a series of immune responses and the production of cytokine storm in the body, which may be associated with the critical condition of Co-19 patient, leading to a severe respiratory failure like ARDS. The $\mathrm{S}$ protein of Coronavirus has been demonstrated to bind to the host cells by ACE2, fusing to the membrane and releasing the viral RNA which is the pathogen-associated molecular patterns (PAMPs) that trigger a downstream cascades of pyrogenic molecules involved in many inflammatory pathways. ${ }^{15}$ Several cytokines and chemokines have been found in the plasma of observed Co-19 patients, including IL-6, IFN- $\gamma$ and TNF- $\alpha$.

Thus, whatever the causes, inflammation and increased capillary permeability are the underlying process in the pathogenesis of ARDS which has been widely described.The first event is the damage of the capillary endothelium and alveolar epithelium in correlation to impaired fluid removal from the alveolar space which result in accumulation of protein-rich fluid inside the alveoli, thereby enhancing a diffuse alveolar damage, with release of pro-inflammatory cytokines, such as Tumor Necrosis Factor (TNF), IL-1 and IL-6. The consequent damaged cellular plasma membrane triggers a self-sustaining alveolar inflammation with surfactant loss production. Pulmonary monocytes and platelets are activated to contain the barrier damage with production of procoagulant factor and platelet degranulation. ${ }^{16,17}$

This event improves vasodilation, increasing blood vessel permeability and permitting the recruitment of other inflammatory cells (neutrophils, macrophages and lymphocytes) from the blood to the site of injury with the phenomenon of leukocyte extravasation. Thus neutrophils are recruited to the lungs by cytokines, become activated and release toxic mediators, such as reactive oxygen species and proteases enhancing the oxidative cell damage. ${ }^{18,19}$

The leucocytes also secrete cytokines and chemokines such as IL-1 and TNF that amplify inflammatory response and promote the recruitment and proliferation of both endothelial cells and fibroblasts to produce fibrotic matrix. This follows the subsequent activation of T cells, which amplify the immune response and promotes the proliferation of fibroblast and their conversion into matrix producing myofribroblasts with the aim to repair the lung damage. An uncontrolled and massive lung involvement of inflammation may lead to ARDS as had occurred in these two cases. Why this comparison between our cases? From a radiation oncologist's point of view, the similarities found in this report could help physicians to adopt several therapeutic novelties of RILI care in the therapeutic approach of this viral pneumonia whose therapy up to now is predominantly supportive amidst the absence of validated antiviral drugs. Among novelties the use of steroids, nebulized heparin, elastase inhibitors like sivelestat sodium, mononuclear autologous cell therapy might be suggested to minimize the acute lung injury. ${ }^{20-22}$

\section{Acknowledgments}

None.

\section{Conflicts of interest}

The authors declare no conflicts of interest.

\section{Funding}

None.

\section{References}

1. Pierrakosa C, Karanikolasb M, Scollettac S, et al. Acute Respiratory Distress Syndrome: Pathophysiology and Therapeutic Options. J Clin Med Res. 2011;4(1):7-16.

2. Bernard GR. Acute respiratory distress syndrome: a historical perspective. Am J Respir Crit Care Med. 2005;172(7):798-806.

3. Chen N, Zhou M, Dong X, et al. Epidemiological and clinical characteristics of 99 cases of 2019 novel coronavirus pneumonia in Wuhan, China: a descriptive study. Lancet. 2020;395(10223):507-513.

4. Hanania NA, Mainwaring W, Ghebre YT, et al. Radiation-Induced Lung Injury Assessment and Management. Chest. 2019:156(1):150-162.

5. Wang D, Hu B, Hu C, et al. Clinical characteristics of 138 hospitalized patients with 2019 novel coronavirus-infected pneumonia in Wuhan, China. JAMA. 2020;323(11):1061-1069.

6. Morgan GW, Pharm B, Breit SN. Radiation and the lung: a reevaluation of the mechanisms mediating pulmonary injury. Int $J$ Radiat Oncol. 1995;31(2):361-369.

7. Ye Z, Zhang Y, Wang Y, et al. Chest CT manifestations of new coronavirus disease 2019(COVID-19): a pictorial review. Eur Radiol. 2020;30:43814389 .

8. Choi YW, Munden RF, Erasmus JJ, et al. Effects of radiation therapy on the lung: radiologic appearances and differential diagnosis. Radiographics. 2004;24(4):985-997.

9. Karpathiou G, Giatromanolaki A, Koukourakis MI, et al. Histological Changes After Radiation Therapy in Patients with Lung Cancer: A Prospective Study. Anticanc Res. 2014;34(6):3119-3124.

10. Sufang T, Xiong Y, Liu H, et al. Pathological study of the 2019 novel coronavirus disease (COVID-19) through postmortem core biopsies. Mod Pathology. 2020;33(6):1007-1014.

11. Xu Z, Shi L, Wang Y, et al. Pathological findings of COVID-19 associated with acute respiratory distress syndrome. Lancet Respir Med. 2020;8(4):420-422

12. Krombach J, Hennel R, Brix $\mathrm{N}$ et al. Priming anti-tumor immunity by radiotherapy: Dying tumor cell-derived DAMPs trigger endothelial cell activation and recruitment of myeloid cells. Oncoimmunol. 2019;8(1):e1523097.

13. Gibson PG, Bryant DH, Morgan GW, et al. Radiation-Induced Lung Injury: A Hypersensitivity Pneumonitis?. Ann Intern Med. 1988;109(4):288-291.

14. Roberts CM, Foulcher E, Zaunders JJ, et al. Radiation pneumonitis: a possible lymphocyte-mediated hypersensitivity reaction. Ann Intern Med. 1993;118(9):696-700.

15. Hamming I, Timens W, Bulthuis ML, et al. Tissue distribution of ACE2 protein, the functional receptor for SARS coronavirus. A first step in understanding SARS pathogenesis. J Pathol. 2004;203(2):631-637.

16. Moine $\mathrm{P}$, McIntyre $\mathrm{R}$, Schwartz $\mathrm{MD}$, et al. NF-kappaB regulatory mechanisms in alveolar macrophages from patients with acute respiratory distress syndrome. Shock. 2000;13(2):85-91.

17. Caudrillier A, Kessenbrock K, Gilliss BM, et al. Platelets induce neutrophil extracellular traps in transfusion-related acute lung injury. J Clin Invest. 2012;122(7):2661-2671.

18. Lierova A, Jelicova M , Nemcova M, et al. Cytokines and radiationinduced pulmonary injuries. $J$ Radiat Res. 2018;59(6):709-753.

19. Cedervall J, Zhang Y, Huang H, et al. Neutrophil Extracellular Traps Accumulate in Peripheral Blood Vessels and Compromise Organ Function in Tumor-Bearing Animals. Cancer Res. 2015;75(13):2653-2662. 
20. Dixon B, Schultz MJ, Hofstra JJ, et al. Nebulized heparin reduces levels of pulmonary coagulation activation in acute lung injury. Crit Care. 2010;14(5):445.

21. Pu S, Wang D, Liu D, et al. Effect of sivelestat sodium in patients with acute lung injury or acute respiratory distress syndrome: a meta-analysis of randomized controlled trials. BMC Pulm Med. 2017;17(1):148.
22. Hayes M, Curley G, Ansari B, et al. Clinical review: Stem cell therapies for acute lung injury/acute respiratory distress syndrome - hope or hype? Crit Care. 2012;16(2):205. 